\title{
First steps: XMP
}

\section{Jason Bright}

is the President/CTO and founder of MediaBeacon Inc. Jason has been creating visionary products in the DAM/MAM space for over a decade. He is both a technical lead and a inventor, bringing his imagination to the customer to solve the most complex industry problems. Recently, he has put his mind to the adoption of the Semantic Web and works toward the day when DAM is core to every computer everywhere.

\section{Keywords: XMP, RDF, XML, Semantic Web, metadata, Adobe}

Abstract Extensible metadata platform (XMP) first step is a discussion of the importance and correctness of XMP and other rich document format-based technology. The article walks the reader through the motivation, evolution and hurdles in XMP. The paper also discusses how MediaBeacon R3volution addressed some of the hurdles and how strong the XMP standard will become.

Journal of Digital Asset Management (2006) 2, 198-202. doi:10.1057/palgrave.dam.3650025

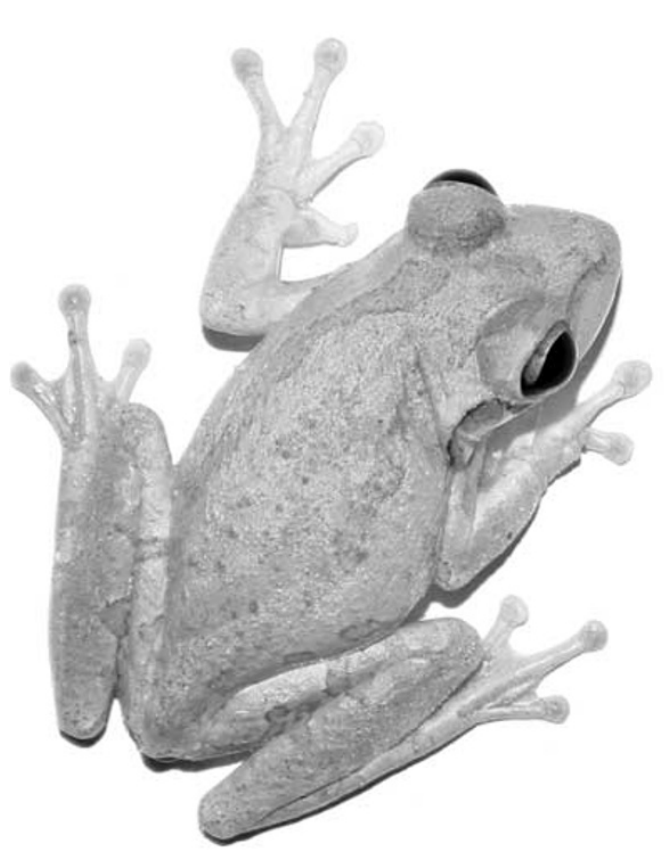

\section{FIRST STEPS: INTRODUCTION}

Not long ago an enterprising young life form crawled his way out of the mud to take a first step upon land and see a new world of possibility.

Quite a bit later, Adobe took a similar first Jason Bright
President/CTO BrighTech Inc.,

212, 3rd Avenue North, Suite 555 ,

Minneapolis, MN 55401, USA

Tel: $+16123170737 \times 100$

E-mail:

jason@Brightech.net step with XMP. This article is a walk in the new landscape that is set to change the internet, digital asset management (DAM) and our lives for the better.

First, there is some background to know about XMP. XMP stands for eXtensible
Metadata Platform and is a standard from Adobe. But that is only half the story. XMP is actually that global internet standard RDF. RDF, or Rich Document Format, is universally used to store information on the web. What is different between XMP and RDF is that Adobe XMP came up with a way to write these data directly into your files, forever gluing your data to your media.

\section{"WHAT IS WRONG WITH SWIMMING?" SOME MAY ASK}

The truth is that there were piranhas in the pond and the pond size limited possible growth. DAM was dying before XMP. In the past, metadata languished exclusively in databases, while the files lay scattered on disk or tape or shelves. The data washed away so easily it was almost worthless. A Post-It just isn't a Post-It if it isn't good and sticky. There was so much reentry and conversion in the world of DAM that it became a joke. People were talking about their data structures in meeting after meeting just to lose that same data a week later.

\section{"WHY WAS IT SO CROWDED?" IS THE NEXT LOGICAL QUESTION ALONG THE PATH}

The truth is, DAM was designed to be used in a single building or a single company. Many of the first standards like IPTC were designed to 
be used by a single group and assumed that things like "transmission name" were important to just everyone. This is called "schema locked," or in other words, the DAM systems force everyone to have the same data about each file. If you don't fit, you are out of luck. The DAM industry realized later that each asset might need different data fields. In response, the DAM industry created the concept of dynamic fields and even dynamic fields per asset. That pond is still not large enough, sadly. Every company has a "size" field for assets. Is "size" a foot, an inch, a millimeter or perhaps an instruction? Is that data in English, Spanish, Chinese or Russian? Each company will have a different interpretation and a different language in our worldwide economy. To cope with this flaw, an entire industry sprang up to do "data mapping" and "internationalization." The DAM pond was too small. The basic idea was crushing the life out of itself and any industry it touched.

\section{THE DAM/MEDIA ASSET MANAGEMENT INDUSTRY WAS STUNTED. WHERE WAS DYNAMIC PUBLISHING? WHERE WAS REPURPOSING? WHERE WAS DATA EXCHANGE? WHERE WERE THE TOOLS?}

After some furious swimming in the muck answers came. But not from the same pond. The answers came from that world-spanning entity the internet. All of these problems were also the rock around the neck of information traveling on the internet. Information travels so fast that there is no way that anyone could possibly "data map" fast enough to meet demands. The internet created RDF, and in turn Adobe snatched the idea and brought it to us as XMP with some darn great glue to ensure it stuck when attached to files.

\section{"WHERE CAN WE GO ON THIS PATH." SOLUTIONS!}

From the liberal use of the past tense you should be able to tell that there is now a set of solutions to solve the DAM/MAM problems.

\section{UNIVERSAL REUSE}

XMP allows every group to add data to every file. Where the old DAM systems used something like an Excel spreadsheet to track each asset and a set of columns shared among all files.

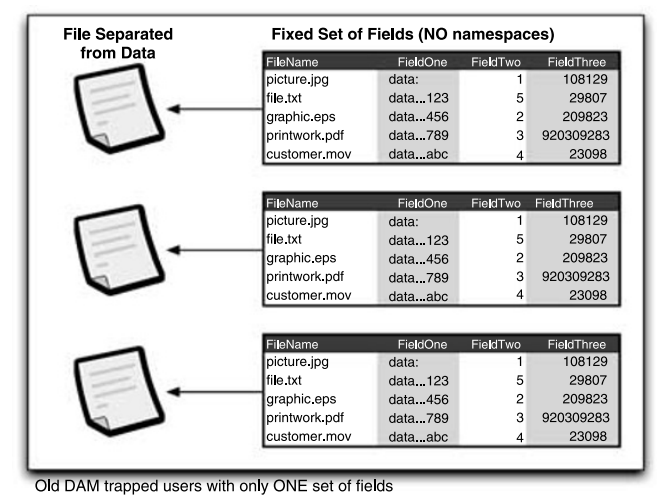

The new namespace-aware XMP systems can allow unlimited fields in unlimited namespaces. Each asset is the head of a train of data with any number of cars following. That means that every field and every language can be included in a file without colliding with any other field in the world.

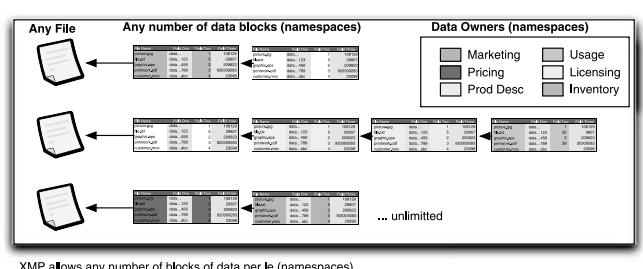

The data has no boundaries. There is no more need to "import" data. All data is always present. Every workflow can continue unfettered by any other workflow in the system.

\section{UNIVERSAL WORKFLOW}

Your workflow can cross continents. Wherever the files go, so does the data. In the old world of DAM, the information crucial to workflow was lost whenever the files left the local server. XMP allows workflows to be continuous even if the files cross continents, are converted or are archived. The database no longer needs to hold every file locally. Things like copyright are not lost. Older systems required mapping to pass data even between departments inside the same building. In place of the long and expensive 
process of importing data from another vendor or source is the simple rule "If you can touch the file you have the data." Millions of dollars of savings for an industry mired in import/ export.

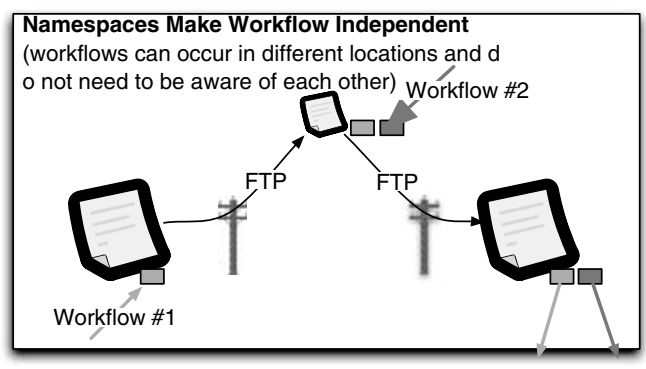

With XMP it is now possible to send one million files to an FTP server and have the recipient correctly process all files based on the data in the files without resorting to using filenames like "NEW_JOB1232232_APPROVE_ JBRIGHT_DUE_TUES.jpg” as was the case when only the filename was sure to be able to carry metadata. With XMP you can truly "Work Locally, Think Globally."

\section{UNIVERSAL SCALE}

The internet could have been a single computer. If the internet were a single computer, we would all talk about how "enterprise" the system was. How "fault tolerant." How "scaleable" the single big computer was. But the internet is not a single computer, it is a network. The internet scales as a group of individual servers scales. The internet is based on cellular growth, allowing it to literally explode on the landscape and fill every nook and every cranny. The internet is enabled by a single standard "HTML" that allows uncounted thousands of servers to send data as you pass from website to website. In the same way, XMP allows the DAM/MAM system to grow in a cellular way. Each DAM node can function in concert with all of the other nodes as files move from location to location. Coupled with the existing internet, DAM becomes universal. There is nothing more scalable. There is nothing more enterprise, and a single computer system, no matter how "enterprise," pales in comparison.

\section{“BEAUTY IS IN THE EYE OF THE BEHOLDER," HOW THE DATA LOOKS MATTERS}

\section{Universal presentation}

Having climbed out of the mire, XMP can strut across the landscape. But data are not valuable if humans don't know where to look for it. Adobe provided the "XMP Custom Template" standard to allow data to be formatted for humans to read. MediaBeacon R3volution, among others, makes use of this Adobe standard to ensure that XMP data is displayed in the same formats no matter if it is on the web, on the desktop, inside Adobe products, inside flash or inside portable document format (PDF). You see the data in the same format that you saw it yesterday. Adobe took a page from Steve Job's book, they made things look standard. Humans can make sense of whatever program they use without relearning how the screen is laid out each time.

\section{"THAT FROG HAS WARTS!" XMP IS NOT WITHOUT ISSUES}

\section{Universal write}

Until now XMP has been hard to write. Adobe published free tools to read XMP, but only a specification on how to write to a few of the graphic file types. Adobe used a rather undocumented method to write XMP to files that could not be stretched to include more information.

MediaBeacon spent two years creating a complete library in 100 per cent Java to read and write XMP to all files on all platforms. MediaBeacon follows the Adobe specification exactly and follows the less-documented method for files that were not specified in the standard. MediaBeacon R3volution uses this library to read and write to all files on OSX, Windows, Linux and even OS9. MediaBeacon follows the XMP standard as defined by Adobe for all files. MediaBeacon uses inline file writing for the formats that allow data to be inserted into the file. Examples of this type of file are PDF, JPG, TIFF, MPG, MOV, AVI, and any other format that can accept additional data without affecting the visual display in any way. Formats that do not allow additional information to be inserted 


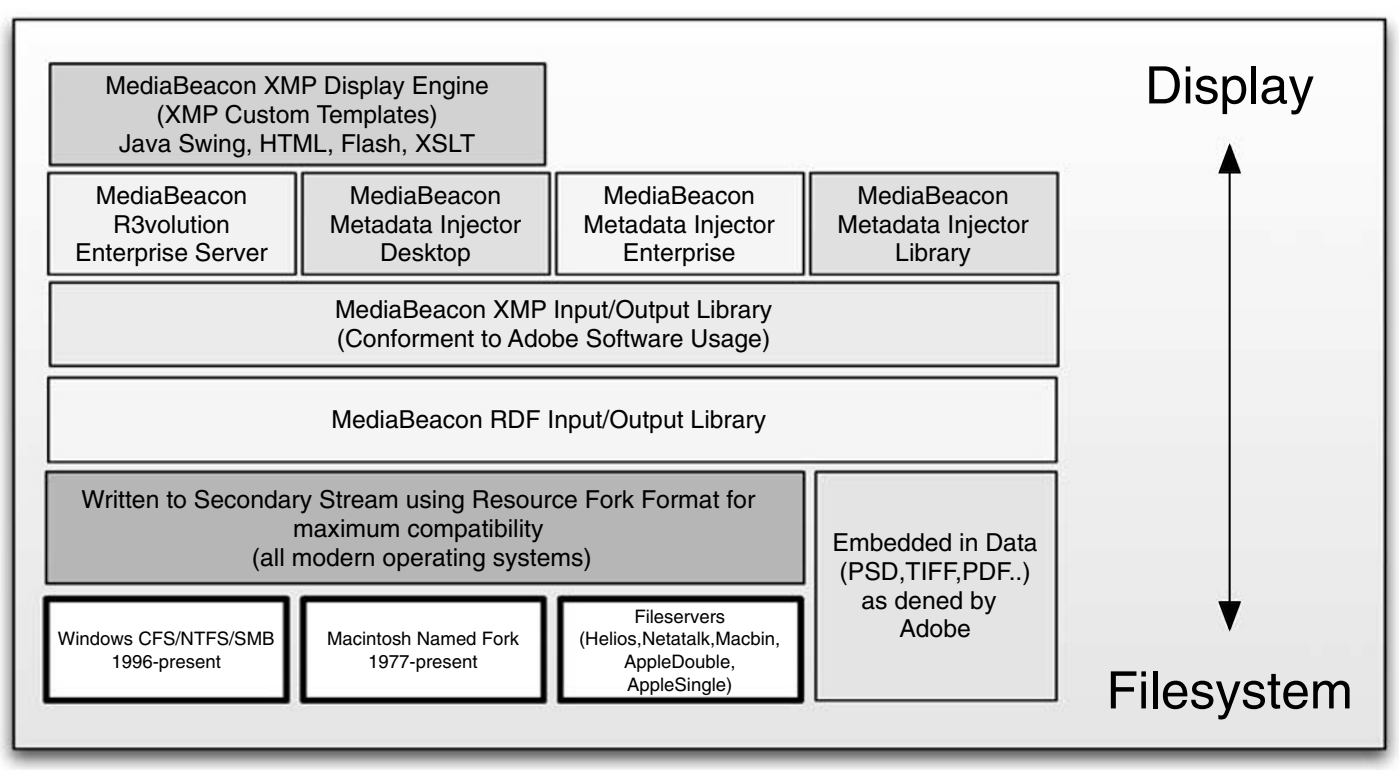

are handled according to the implicit standard used by Adobe. These files have information written to the additional file streams in resource format. Additional streams are file components that are managed as a part of a file transparently throughout modern operating systems. Multiple streams are a part of Windows, OSX, Linux, and Solaris. All modern platforms are capable of storing information in this way. Adobe took advantage of this to bring XMP to files not able to be extended or unknown files. Adobe may also write "sidecar" files which create this same data as an external component in a very few cases. MediaBeacon further adds support the "XMP Custom Templates" to allow all data to be displayed in the same look-and-feel in all cases. MediaBeacon XMP looks exactly like the panels found in any Adobe product. This allows users to immediately find the data they need without having to read every caption on every data page. MediaBeacon also provides this library to the IDEAlliance for distribution as part of several metadata initiatives, and this library is available at no charge for noncommercial use. Enterprise customers can now use this library to interact with any server that supports Java.

Adobe also has a soon to be released XMP toolkit in $\mathrm{C}++$ that will support read and write for a subset of the files supported by MediaBeacon R3volution. The Adobe library is
100 per cent compatible with MediaBeacon's library for the file types Adobe specifies.

\section{Universal security}

Another prominent wart on XMP is the complete lack of security. XMP is written by almost every Adobe application, but is always completely visible. In the case of PDFs of medical or financial documents, this can mean a SOX or HIPPA subpoena or worse.

In the case of customer data, it can mean lost business. MediaBeacon solves this problem and allows each field to be encrypted with a separate enterprise encryption key. The data are 100 per cent attached to each file, but is also enterprise-grade encrypted. Only partners who hold the keys to the data can decrypt it. This simple feature should be a must to anyone who is considering using XMP or any of its kin (below) for professional use.

\section{"Lions and tigers and bears." Relatives of XMP}

Adobe wasn't the only group of geniuses that realized XMP was the right answer. Almost every industry you see has some form of RDF as well. XMP is cousins with MXF, RSS, MPEG7 and many other industry standards. The overwhelming success of the RDF format under each of these names ensures XMP's success. Adobe's direction is not proprietary. It is the right answer. 


\section{“WHERE IS ALL THIS WALKING LEADING?" WHAT DOES THIS MEAN TO YOU?}

\section{Digital rights management (DRM)}

Currently lawyers extract thousands of dollars of penalties and fees from publishing companies for honest and unintentional theft of media. Complex contracts simply can't be followed at the current production rates and businesses have adopted a duck and cover strategy. With XMP, the license for every file everywhere can be known and checked at use. Whether computer enforced or simply part of the approval process, the risk is gone.

\section{Dynamic publishing}

Finally any product picture that finds its way into a database can contain the English, German and French descriptions of the products with pricing, inventory and reference uniform resource locators. Data are in a standard format and a few seconds after it is received it can be put to use. Dynamic publishing can exist without Excel spreadsheets.

\section{Enterprise content management (ECM)}

ECM systems barely handle image data and simply ignore most of the workflow of the graphics industry. Working with XMP means that the ECM systems can track information through the graphic workflows. ECM can both deliver the process control and allow you to use current graphic workflows transparently. Prepress can find its way to the enterprise workflow. Creative professionals can finally be central to the everyday lifecycle of their industry instead of relegated to make pretty work.

\section{Distributed search}

As should every other tool, MediaBeacon R3volution uses the XMP format to integrate into the desktop search tools available now from Apple (Spotlight) and the upcoming tools from Microsoft (Vista, Index Server).

\section{Best of breed business}

No single software company can predict the needs of any large set of businesses. Each business must be free to select the best of breed solutions for their unique needs. $\mathrm{XMP}$ and RDF allow each business to select the software that best fits their needs. Instead of being trapped inside a single vendor's solution, the XMP standard sets you free.

These boots were made for walking.... Are you ready boots?... Start walkin! 